\title{
CORRESPONDENCE
}

\section{Pseudo B-line in pneumothorax: first look at the patient. Author's reply}

\author{
Adrian Wong ${ }^{1 *}$ (D) Olusegun Olusanya ${ }^{2}$ and Alberto Goffi ${ }^{3}$
}

(0) 2020 Springer-Verlag GmbH Germany, part of Springer Nature

\section{Dear Editor,}

We read with great interest the article by Idei and Colleagues [1] highlighting the importance of scrutinizing visualised B-lines before committing to critical decision-making such as the exclusion of pneumothorax. It is a timely reminder that appropriate training and quality control of images is crucial especially given the rapid expansion in the use of lung ultrasound during the coronavirus disease 2019 (COVID-19) pandemic.

However, there are a number of other issues pertinent to the case that we would like the authors and readers to consider.

Firstly, as with any other test, test accuracy needs to be incorporated with clinical context (i.e. pre-test probability), as explained by Bayes' theorem and previously discussed in this journal [2]. Although no detailed clinical information was presented, the authors mention that the patient had a refractory pneumothorax. Moreover, from the image of the patient provided it is clear he has had a number of ICU interventions (tracheostomy, suture on chest, chest tube thoracostomy in place-also seen on the CT scan, etc.). All this information taken together, in our opinion, would have suggested a high pre-test probability of residual pneumothorax. Even assuming the best-reported sensitivity (90\%) for the detection of pneumothorax on lung ultrasound, we would still have expected a significant false-negative rate [3].

Secondly, the Authors mention that the comet-tail artifact in their patient originated from "between parietal pleura and its outer layer tissue". It is unclear what is meant by this term. In previously published manuscripts, "pseudo B-lines" originated from the pleura but also subcutaneous emphysema $[4,5]$. Indeed, on closer inspection of the images provided, both the CT scan and B-mode lung ultrasound suggest the presence of pleural thickening/plaque (Fig. 1).

Finally, the Authors state that the comet-tail artifact in their patient showed "synchronized movement with chest wall motion instead of lung sliding" and "with breathing". We agree that the vertical artifacts demonstrated here should be synchronous with active movements of the chest wall since they originate from within the chest wall. However, as lung sliding occurs when parietal and visceral pleura are in contact and freely moving, presence of pleural adhesions could create similar findings (i.e., lack of sliding but the presence of vertical artifact/B-lines). 

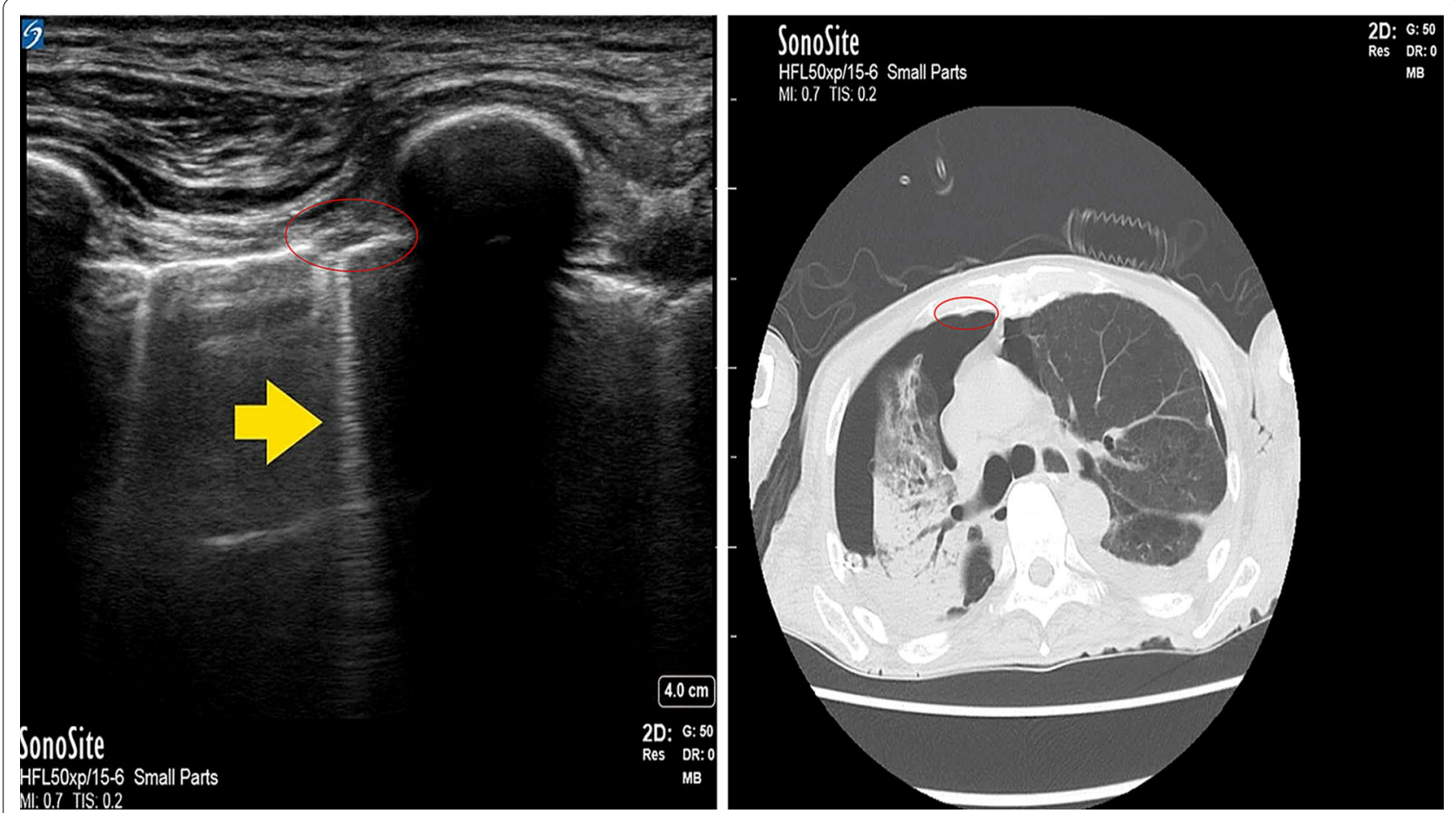

Fig. 1 Lung ultrasound and chest CT with areas of pleural thickening circled

\section{Author details}

${ }^{1}$ Consultant Critical Care, King's College Hospital, London, UK. ${ }^{2}$ Barts Health NHS Trust, London, UK. ${ }^{3}$ Department of Critical Care Medicine and Li Ka Shing Knowledge Institute, St. Michael's Hospital Toronto, Toronto, Canada.

\section{Compliance with ethical standards}

\section{Conflicts of interest}

The authors do not have any conflict of interest to declare.

\section{Publisher's Note}

Springer Nature remains neutral with regard to jurisdictional claims in published maps and institutional affiliations.

Accepted: 15 September 2020

Published online: 8 October 2020

\section{References}

1. Idei M, Seino Y, Nomura T (2020) Pseudo B-line in pneumothorax. Intensive Care Med 46:1468-1469. https://doi.org/10.1007/s00134-019-05860 $-2$

2. Mayo PH, Copetti R, Feller-Kopman D et al (2019) Thoracic ultrasonography: a narrative review. Intensive Care Med 45:1200-1211. https://doi. org/10.1007/s00134-019-05725-8

3. Hew M, Tay TR (2016) The efficacy of bedside chest ultrasound: from accuracy to outcomes. Eur Resp Rev 25:230-246

4. Laursen CB, Graumann O, Davidsen JR, Madsen PH (2014) Pitfall in lung ultrasound: 'pseudo B-line' seen in both hydropneumothorax and in a cup of coffee. BMJ Case Rep. https://doi.org/10.1136/bcr-2013-201341

5. Volpicelli G, Garofalo G, Lamorte A, Frascisco MF (2012) Images in emergency medicine. Young man with left thoracic pain. Recurrent pneumothorax after failed pleurodesis. Ann Emerg Med 60(2):3-4. https ://doi.org/10.1016/j.annemergmed.2012.03.002 